\title{
STRUCTURAL BASIS OF TRANSCRIPTION INITIATION
}

\author{
Yu Zhang ${ }^{1}$, Yu Feng ${ }^{1}$, Sujoy Chatterjee ${ }^{1}$, Steve Tuske ${ }^{2}$, Mary X. Ho$^{2}$, Eddy Arnold ${ }^{2}$, and \\ Richard H. Ebright ${ }^{1,{ }^{,}}$ \\ ${ }^{1}$ Howard Hughes Medical Institute, Waksman Institute, and Department of Chemistry and \\ Chemical Biology, Rutgers University, Piscataway NJ 08854, USA \\ ${ }^{2}$ Center for Advanced Biotechnology and Medicine and Department of Chemistry and Chemical \\ Biology, Rutgers University, Piscataway NJ 08854, USA
}

\begin{abstract}
During transcription initiation, RNA polymerase (RNAP) binds and unwinds promoter DNA to form an RNAP-promoter open complex. We have determined crystal structures at 2.9 and $3.0 \AA$ resolution of functional transcription initiation complexes comprising Thermus thermophilus RNA polymerase, $\sigma^{\mathrm{A}}$, and a promoter DNA fragment corresponding to the transcription bubble and downstream dsDNA of the RNAP-promoter open complex. The structures show that $\sigma$ recognizes the -10 element and discriminator element through interactions that include the unstacking and insertion into pockets of three DNA bases, and that RNAP recognizes the $-4 /+2$ region through interactions that include the unstacking and insertion into a pocket of the +2 base. The structures further show that interactions between $\sigma$ and template-strand ssDNA pre-organize template-strand ssDNA to engage the RNAP active center.
\end{abstract}

In transcription initiation, RNA polymerase (RNAP), together with at least one transcription initiation factor, binds to promoter DNA to yield an RNAP-promoter closed complex (RPc) and then unwinds $\sim 12$ bp of promoter DNA to form a "transcription bubble" and yield an RNAP-promoter open complex (RPo; 1). RPo is the critical, catalytically competent, intermediate in transcription initiation, and modulation of the formation, stability, and activity of RPo is an important means of regulation of gene expression (1). Structural models of RPo have been generated based on information from electron microscopy, fluorescence-resonance-energy-transfer measurements, and protein-DNA crosslinking (2-9). However, no high-resolution structural information for a functional, promoter-dependent, initiation-factor-dependent RPo previously has been reported.

Here, we report crystal structures of a bacterial RPo and a bacterial RPo in complex with a ribodinucleotide primer at 2.9 and $3.0 \AA$ resolution, respectively. The results define the interactions of RNAP and the transcription initiation factor $\sigma$ with the nontemplate and template strands of the transcription-bubble in RPo, the interactions of RNAP with downstream dsDNA in RPo, and the RNAP clamp conformation in RPo.

To obtain a structure of RPo, we used a synthetic nucleic-acid scaffold corresponding to the transcription bubble and downstream dsDNA of RPo (10; Fig. S1). The top strand comprised a 14-nt ssDNA tail containing a consensus promoter -10 element (11) and consensus discriminator element (12-13), followed by a 13-nt duplex-forming segment. The bottom strand comprised a non-complementary 6-nt ssDNA tail, followed by a 13-nt duplexforming segment. The scaffold was functional in RNAP-DNA complex formation (Fig. S1E) and in de novo transcription initiation with position +1 as the transcription start site (Fig.

*Corresponding author: Phone: 848-445-5179 (RHE) Fax: 732-445-5735 (RHE). ebright@ waksman.rutgers.edu. 
S1F). Published work indicates that scaffolds of this form recapitulate all functional properties of the transcription bubble and downstream dsDNA of RPo (14).

We prepared complexes of the scaffold with Thermus thermophilus RNAP holoenzyme containing $\sigma^{\mathrm{A}}$, identified crystallization conditions by use of robotic crystallization trials, grew crystals, collected data at a synchrotron, solved the structure by molecular replacement, and refined the structure, yielding a structure of RPo with a resolution of $2.9 \AA$ and $\mathrm{R}_{\text {free }}$ of 0.226 (Fig. S1). Electron-density maps showed unambiguous density for nontemplate-strand nucleotides -12 to +12 , template-strand nucleotides -4 to $+12,3139$ RNAP residues, and $346 \sigma$ residues, and showed density for template-strand nucleotides +1 and +2 in the RNAP active-center " $\mathrm{i}$ " and " $\mathrm{i}+1$ " sites. Analysis of crystals obtained using a derivative of the scaffold containing $5-\mathrm{BrU}$ in place of $\mathrm{T}$ at template-strand position +1 showed a single peak of $\mathrm{Br}$ anomalous difference density at the expected position, confirming that RNAP interacts with the scaffold in the crystals in a single translocational register, and confirming that the translocational register places template-strand nucleotides +1 and +2 in the RNAP active-center "i site" and "i+1 site" (Fig. S2).

To obtain a structure of RPo in complex with a ribodinucleotide primer, we used an analogous nucleic-acid scaffold containing $\mathrm{GpA}$, a ribodinucleotide complementary to template-strand positions -1 and +1 (Figs. 1A, S3). Biochemical experiments verified that this scaffold is functional in primer-dependent transcription initiation (Fig. S3E-F). Crystals were prepared, and data were collected and processed as above, yielding a structure of RPoGpA with a resolution of $3.0 \AA$ and $R_{\text {free }}$ of 0.247 (Figs. 1A-B, S3). Protein-DNA interactions were essentially identical in the structures of RPo and RPo-GpA (Fig. S4).

The structures show that RNAP and $\sigma$ recognize promoter elements through sequencespecific interactions with transcription-bubble nontemplate-strand ssDNA (Figs. 1-3). $\sigma$ makes sequence-specific interactions with the upstream part of the transcription-bubble nontemplate strand (positions -12 to -4 ; Figs. $1 \mathrm{~A}-\mathrm{C}, 2$ ), and RNAP makes sequencespecific interactions with the downstream part of the transcription-bubble nontemplate strand (positions -4 to +2 ; Figs. 1A-C, 3). $\sigma$ interacts with the promoter -10 -element nontemplate strand through a deep, L-shaped groove that runs across $\sigma$ region $2(\sigma \mathrm{R} 2)$ and part of $\sigma$ region $1.2(\sigma \mathrm{R} 1.2$; Figs. $1 \mathrm{~A}-\mathrm{C}, 2 \mathrm{~A})$.

$\sigma$ interacts with all six nucleotides of the -10 element and potentially interacts with bases of five nucleotides of the -10 element (positions $-12,-11,-9,-8$, and -7 ; Figs. 1A-C, 2A, S5-S6). The interactions with bases involve $\sigma$ residues L110, E116, N383, R385, L386, K418, F419, E420, R423, Y425, S428, T429, Y430, T432, W433, and R436 (residues numbered as in Escherichia coli RNAP and $\sigma^{70}$ ). Alanine substitutions of these residues result in defects in transcription, indicating that these residues are functionally significant (Fig. 2B). Model building indicates that $\sigma$ residues Q437 and T440 could contact a nucleotide base-paired to nontemplate-strand position -12, providing a structural explanation for results indicating these residues are important for sequence recognition at position -12 (15-16; Figs. 2A, S5). The interactions between $\sigma$ and the -10 element in RPo and RPoGpA are essentially identical to those observed in a crystal structure of a $\sigma \mathrm{R} 2$ fragment bound to a -10 element ssDNA oligonucleotide (17-18).

$\sigma$ interacts with the promoter discriminator-element nontemplate strand through a shallow groove that runs across the face of $\sigma \mathrm{R} 1.2$ (Figs. 1A, 2C). $\sigma$ interacts with bases of three nucleotides of the discriminator element (positions -6 through -4 ; Figs. 1A, 2C, S7). The interactions involve $\sigma$ residues D96, V98, R99, M102, R103, M105, G106, and R385.

Alanine substitutions of these residues result in defects in transcription (Fig. 2D). $\sigma$ residue M102 makes direct van-der-Waals contact with the base at nontemplate-strand position -5 , 
providing a structural explanation for the observation that $\sigma$ M102 can be crosslinked to this base (19; Figs 1A, 2C, S7B).

A striking feature of the interactions between $\sigma$ and the -10 and discriminator elements is that $\sigma$ unstacks three DNA bases, flips them out of base stacks, and inserts them into pockets formed by residues of $\sigma$ (Figs. 1, 2, S6A, C, S7A). $\sigma \mathrm{R} 2$ unstacks, flips, and inserts into a deep pocket the adenine base at the second position of the -10 element (A-11; Figs. 2A, E, S6A). Within this deep pocket, A-11 makes stacking interactions with one aromatic amino acid ( $\sigma$ Y430), makes edge-edge interactions with two aromatic amino acids ( $\sigma$ Y419 and Y425), and makes H-bonds between base Watson-Crick atoms and two amino-acid backbone atoms ( $\sigma$ K418 and Y419), enabling unambiguous sequence read-out (Fig. S6A). In an analogous manner, $\sigma \mathrm{R} 2$ unstacks, flips, and inserts into a deep pocket the thymine base at the sixth position of the -10 element (T-7; Figs. 2A, C, F, S6C) and $\sigma \mathrm{R} 1.2$ unstacks, flips, and inserts into a deep pocket the guanine base at the first position of the discriminator element (G-6; Figs. 2C, F, S7A). The insertion of flipped-out bases into pockets provides an effective means to read sequence, since it enables contacts with essentially all atoms of the bases. This mode of interaction accounts for the fact that A-11 and T-7 are the most important positions of the -10 element (11). The insertion of flipped-out bases into pockets also provides an effective means to use binding energy to drive DNA unwinding, since this mode of interaction can occur only when DNA is unwound. This mode of interaction accounts for the ability of $\sigma$ to facilitate promoter unwinding.

RNAP core enzyme interacts with transcription-bubble nontemplate-strand positions -4 to +2 , which we term the "core recognition element" (CRE; Figs. 1A, C, 3), and which we show to be a sequence-specific promoter element (see below). RNAP core enzyme interacts with bases of five of six nucleotides of the CRE (positions $-4,-3,-2,+1$, and +2 ; Figs. 1A, 3A, S8). The interactions involve RNAP $\beta$-subunit residues R151, W183, D199, R371, R394, I445, D446, R451, L538, and V547. Alanine substitutions of all except one of these residues result in defects in transcription (Fig. 3B). The interactions account for the previously observed crosslinking of nontemplate-strand positions -4 though +2 to $\beta$ residues 84-642 (2).

The interactions between RNAP and the most-downstream nucleotide of the transcriptionbubble nontemplate strand, $\mathrm{G}+2$, are especially noteworthy. RNAP unstacks the $\mathrm{G}+2$ base, flips it out of the base stacks, and inserts it into a deep pocket formed by RNAP $\beta$ subunit (" $\beta$ pocket"), in a manner analogous to the manner in which $\sigma$ interacts with A-11, T-7, and G-6 (Fig. 3A, C, S8C). Six residues of the $\beta$ pocket make van-der-Waals interactions with the $\mathrm{G}+2$ base ( $\beta$ R151, I445, D446, R451, L538, and V547), and two residues of the $\beta$ pocket make H-bonds with Watson-Crick atoms of the G+2 base ( $\beta$ D446 and R451; Fig. S8C). The structures suggest that the interactions between RNAP and G+2 are sequencespecific (Fig. S8C), and equilibrium binding and kinetic experiments confirm that the interactions between RNAP and G+2 are sequence-specific (Figs. 3D-E, S9). RNAP exhibits an $\sim 5$-fold lower equilibrium dissociation constant, and an $\sim 5$-fold lower off-rate, for a promoter derivative having $\mathrm{G}$ at nontemplate-strand position +2 than for promoter derivatives having $\mathrm{A}, \mathrm{T}, \mathrm{C}$, or an abasic site at nontemplate-strand position +2 (Figs. 3D-E, S9). In principle, the sequence-specific interactions between RNAP and nontemplate-strand $\mathrm{G}+2$ shown here to occur in transcription initiation complexes may occur also in transcription elongation complexes. More than thirty crystal structures of elongation complexes have been determined to date (Fig. S10). However, only one includes nontemplate-strand position +2: namely, PDB 3PO2, a structure of a yeast RNAPII backtracked and arrested elongation complex (20). Strikingly, in this structure, although not noted in the publication on this structure, the nontemplate-strand position +2 base is inserted into a pocket formed by RNAPII residues equivalent to $\beta$ R151, $\beta \mathrm{I} 445, \beta \mathrm{D} 446, \beta \mathrm{R} 451$, 
$\beta \mathrm{L} 538$, and $\beta \mathrm{V} 547$, adopting a conformation similar to, and making interactions similar to, $\mathrm{G}+2$ in the structures of RPo and RPo-GpA (Fig. S10). This observation, together with other observations (21) suggests that the RNAP-DNA interactions that mediate recognition of G +2 in initiation complexes may occur also in elongation complexes, where they may influence sequence-dependent translocational bias (22) and sequence-dependent pausing (23). This observation further suggests that these RNAP-DNA interactions may be made not only by bacterial RNAP but also by eukaryotic RNAPII.

The interaction between RNAP and the adjacent nucleotide of the transcription-bubble nontemplate strand, $\mathrm{T}+1$, also is noteworthy. RNAP $\beta$-subunit residue W183 makes an aromatic-aminoacid/base stacking interaction with $\mathrm{T}+1$ (Fig. 3C, S8B). This interaction forces the unstacking of $\mathrm{T}+1$ and $\mathrm{G}+2-$--making $\mathrm{G}+2$ available to interact with the $\beta$ pocket-and also likely nucleates or stabilizes the stacking of nucleotides at nontemplate-strand positions -5 through +1 (Fig. S8B).

Although it has been established that RNAP-CRE interactions involving position +2 are sequence-specific (Figs. 3D-E, S9), it remains to be determined whether RNAP-CRE interactions involving positions -4 through +1 are sequence-specific. We conclude that RNAP-CRE interactions contribute to sequence-specific promoter recognition, and we propose that RNAP-CRE interactions also contribute to the formation and maintenance of the transcription bubble. It seems likely that RNAP-CRE interactions enable RNAP to assist $\sigma$ in promoter unwinding during $\sigma$-dependent initiation, and enable RNAP to perform promoter unwinding during $\sigma$-independent initiation.

The structures also show that RNAP and $\sigma$ "pre-organize" the transcription-bubble template strand and downstream dsDNA (Fig. 4A-B). The transcription-bubble template strand in the structures adopts the same A-form helical conformation, and makes the same interactions with the RNAP active-center " $i$ " and " $i+1$ " sites, as in an elongation complex (Fig. 4A). The downstream dsDNA and the ribonucleotide primer GpA in the structures also exhibit the same conformations and interactions as the downstream dsDNA and the $3^{\prime}$ ribonucleotides of the RNA product in an elongation complex (Fig. 4A). We conclude that a promoterdependent, initiation-factor-dependent transcription initiation complex is pre-organized to proceed to transcription elongation without major changes in the conformation or interactions of the transcription-bubble template strand, downstream dsDNA, and RNA. The pre-organization of the template strand in the promoter-dependent, initiation-factordependent initiation complex is in contrast to the situation in promoter-independent, initiation-factor-independent initiation complexes, in which template-strand ssDNA is disordered (24), or all but $5 \mathrm{nt}$ of template-strand ssDNA is disordered (25), except in the presence of $\geq 4 \mathrm{nt}$ of RNA. The presence of the initiation factor appears to account for the difference. The initiation factor $\sigma$ makes direct interactions with the template strand that preorganize the template strand. The segment of $\sigma$ region $3.2(\sigma \mathrm{R} 3.2)$ comprising $\sigma$ residues $510-522-$-the " $\sigma$ finger"--penetrates the RNAP active-center cleft, occupies part of the region occupied by RNA in a transcription elongation complex, makes an aromatic-aminoacid/base edge-edge interaction with the template-strand base at position -4 , and makes four H-bonds with Watson-Crick atoms of the template-strand bases at positions -4 and -3 (Fig. $4 \mathrm{~B}, \mathrm{~S} 11$ ). The interactions with DNA bases at template-strand positions -4 and -3 involve $\sigma$ residues D514, D516, D517, and F522. Alanine substitutions of these residues result in defects in transcription (Fig. 4C). The interactions between the $\sigma$ finger and the template strand constrain the template strand to adopt an A-form helical conformation and buttress the template strand to engage the RNAP active center in a manner compatible with binding of initiating NTPs. These interactions provide a structural explanation for the ability of the $\sigma$ finger to facilitate the binding of initiating NTPs (26-27). The interactions between the $\sigma$ finger and the template strand must be disrupted, and the $\sigma$ finger must be displaced, in 
order to synthesize $>4 \mathrm{nt}$ of RNA. The need to disrupt these interactions and displace the $\sigma$ finger provides a structural explanation for effects of the $\sigma$ finger on abortive initiation (2627).

The structures also define the conformational state of the RNAP clamp--the movable wall of the RNAP active-center cleft (28)--in the transcription initiation complex (Fig. 4D-E). The clamp is closed by $\sim 11^{\circ} \mathrm{C}$ relative to in the crystal structure of RNAP holoenzyme (Fig. 4D) and exhibits the same conformation as in the crystal structure of the elongation complex (Fig. 4E), consistent with fluorescence-resonance-energy-transfer results indicating that the clamp closes upon formation of RPo and remains closed during elongation (28). The finding that clamp conformations are the same in the initiation complex and the elongation complex provides further evidence that the initiation complex is pre-organized to proceed to elongation.

The structures of RPo and RPo-GpA determined in this work reveal how RNAP and $\sigma$ interact with the transcription-bubble nontemplate strand to accomplish promoter recognition and promoter unwinding, and reveal how RNAP and $\sigma$ interact with the transcription-bubble template strand and downstream dsDNA to pre-organize the transcription initiation complex for subsequent reactions. The structures provide a foundation for understanding transcription initiation and transcriptional regulation.

\section{Supplementary Material}

Refer to Web version on PubMed Central for supplementary material.

\section{Acknowledgments}

We thank the Brookhaven National Synchrotron Light Source and Cornell High Energy Synchrotron Source for beamline access, the Argonne Photon Source CCP4 School for training; and S. Borukhov, P. deHaseth, K. Kuznedelov, L. Minakhin, K. Severinov, and D. Temiakov for plasmids and discussion. This work was funded by NIH grants GM41376 and AI072766 and an HHHMI Investigatorship to RHE. PDB accession codes are 4G7H, $4 \mathrm{G} 7 \mathrm{Z}$, and $4 \mathrm{G} 7 \mathrm{O}$.

\section{Abbreviations}

$\begin{array}{ll}\text { RNAP } & \text { RNA polymerase } \\ \mathbf{R P}_{\mathbf{c}} & \text { RNAP-promoter closed complex } \\ \mathbf{R P}_{\mathbf{0}} & \text { RNAP-promoter open complex }\end{array}$

\section{REFERENCES AND NOTES}

1. Saecker R, Record MJ, Dehaseth P. Mechanism of bacterial transcription initiation: RNA polymerase-promoter binding, isomerization to initiation-competent open complexes, and initiation of RNA synthesis. J Mol Biol. 2011; 412:754. [PubMed: 21371479]

2. Naryshkin N, Revyakin A, Kim Y, Mekler V, Ebright R. Structural organization of the RNA polymerase-promoter open complex. Cell. 2000; 101:601. [PubMed: 10892647]

3. Mekler V, et al. Structural organization of bacterial RNA polymerase holoenzyme and the RNA polymerase-promoter open complex. Cell. 2002; 108:599. [PubMed: 11893332]

4. Hudson B, et al. Three-dimensional EM structure of an intact activator-dependent transcription initiation complex. Proc Natl Acad Sci USA. 2009; 106:19830. [PubMed: 19903881]

5. Murakami K, Masuda S, Campbell E, Muzzin O, Darst S. Structural basis of transcription initiation: an RNA polymerase holoenzyme-DNA complex. Science. 2002; 296:1285. [PubMed: 12016307] 
6. Liu X, Bushnell D, Wang D, Calero G, Kornberg R. Structure of an RNA polymerase II-TFIIB complex and the transcription initiation mechanism. Science. 2009; 327:206. [PubMed: 19965383]

7. Kostrewa D, et al. RNA polymerase II-TFIIB structure and mechanism of transcription initiation. Nature. 2009; 462:323. [PubMed: 19820686]

8. Treutlein B, et al. Dynamic architecture of a minimal RNA polymerase II open promoter complex. Mol Cell. 2012; 46:136. [PubMed: 22424775]

9. Grünberg S, Warfield L, Hahn S. Architecture of the RNA polymerase II preinitiation complex and mechanism of ATP-dependent promoter opening. Nature Structl Mol Biol. 2012; 19:788-796.

10. Materials and methods are available as supplementary materials on Science Online.

11. Shultzaberger R, Chen Z, Lewis K, Schneider T. Anatomy of Escherichia coli $\sigma^{70}$ promoters. Nucl Acids Res. 2007; 35

12. Feklistov A, et al. A basal promoter element recognized by free RNA polymerase sigma subunit determines promoter recognition by RNA polymerase holoenzyme. Mol Cell. 2006; 23:97. [PubMed: 16798040]

13. Haugen $\mathrm{S}$, et al. rRNA Promoter regulation by nonoptimal binding of $\sigma$ region 1.2: an additional recognition element for RNA polymerase. Cell. 2006; 125:1069. [PubMed: 16777598]

14. Mekler V, Minakhin L, Severinov K. A critical role of downstream RNA polymerase-promoter interactions in the formation of initiation complex. J Biol Chem. 2011; 286:22600. [PubMed: 21525530]

15. Siegele D, Hu J, Walter W, Gross C. Altered promoter recognition by mutant forms of the sigma-70 subunit of Escherichia coli RNA polymerase. J Mol Biol. 1989; 206:591. [PubMed: 2661828]

16. Waldburger C, Gardella T, Wong R, Susskind M. Changes in conserved region 2 of Escherichia coli $\sigma^{70}$ affecting promoter recognition. J Mol Biol. 1990; 215:267. [PubMed: 2213883]

17. Feklistov A, Darst S. Structural basis for promoter -10 element recognition by the bacterial RNA polymerase sigma subunit. Cell. 2011; 147:1257. [PubMed: 22136875]

18. Liu X, Bushnell D, Kornberg R. Lock and key to transcription: $\sigma$-DNA interaction. Cell. 2011; 147:1218. [PubMed: 22153066]

19. Haugen S, Ross W, Manrique M, Gourse R. Fine structure of the promoter- $\sigma$ region 1.2 interaction. Proc Natl Acad Sci USA. 2008; 105:3292. [PubMed: 18287032]

20. Cheung A, Cramer P. Structural basis of RNA polymerase II backtracking, arrest and reactivation. Nature. 2011; 471:249. [PubMed: 21346759]

21. Kireeva M, Domecq C, Coulombe B, Burton Z, Kashlev M. Interaction of RNA polymerase II fork loop 2 with downstream non-template DNA regulates transcription elongation. J Biol Chem. 2011; 286:30898. [PubMed: 21730074]

22. Hein P, Palangat M, Landick R. RNA transcript $3^{\prime}$-proximal sequence affects translocation bias of RNA polymerase. Biochem. 2011; 50:7002. [PubMed: 21739957]

23. Landick R. Transcriptional pausing without backtracking. Proc Natl Acad Sci U S A. 2009; 106:8797. [PubMed: 19470457]

24. Liu, X.; Bushnell, D.; Silva, D.; Huang, X.; Kornberg, R. Initiation complex structure and promoter.

25. Cheung A, Sainsbury S, Cramer P. Structural basis of initial RNA polymerase II transcription. EMBO J. 2011; 30:4755. [PubMed: 22056778]

26. Murakami K, Masuda S, Darst S. Structural basis of transcription initiation: RNA polymerase holoenzyme at 4 Å resolution. Science. 2002; 296:1280. [PubMed: 12016306]

27. Kulbachinskiy A, Mustaev A. Region 3.2 of the $\sigma$ subunit contributes to the binding of the $3^{\prime}$ initiating nucleotide in the RNA polymerase active center and facilitates promoter clearance during initiation. J Biol Chem. 2006; 281:18273. [PubMed: 16690607]

28. Chakraborty A, et al. Opening and closing of the bacterial RNA polymerase clamp. Science. 2012; 337:591-595. [PubMed: 22859489]

29. Vassylyev D, Vassylyeva M, Perederina A, Tahirov T, Artsimovitch I. Structural basis for transcription elongation by bacterial RNA polymerase. Nature. 2007; 448:157. [PubMed: 17581590] 
30. Vassylyev D, et al. Crystal structure of a bacterial RNA polymerase holoenzyme at $2.6 \AA$ resolution. Nature. 2002; 417:712. [PubMed: 12000971] 

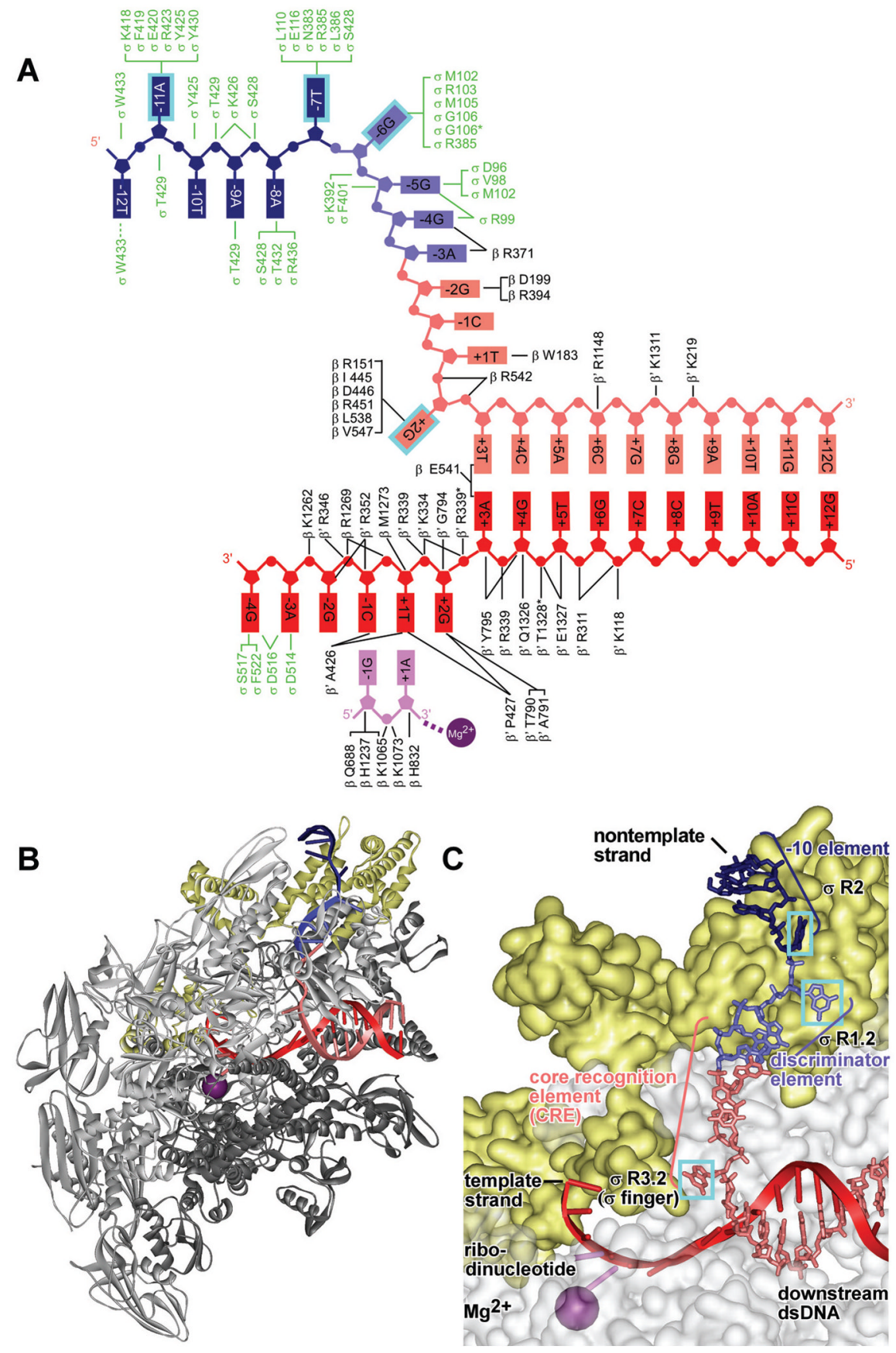

Fig. 1. Structure of RPo-GpA

(A) Summary of protein-nucleic-acid interactions. Black residue numbers and lines, interactions by RNAP; green residue numbers and lines; interactions by $\sigma$; blue, -10 element of DNA nontemplate strand; light blue, discriminator element of DNA nontemplate strand; pink, rest of DNA nontemplate strand; red, DNA template strand; magenta, GpA; violet, active-center $\mathrm{Mg}^{2+}$; asterisks, water-mediated interactions; cyan boxes, bases unstacked and inserted into pockets. Residues are numbered as in E. coli RNAP and $\sigma^{70}$. (B) Overall structure (RNAP $\beta^{\prime}$ non-conserved domain omitted for clarity). RNAP, gray; $\sigma$, yellow. Other colors as in A. (C) Interactions of RNAP and $\sigma$ with transcription-bubble 
nontemplate strand, transcription-bubble template strand, and downstream dsDNA (RNAP $\beta$ subunit and $\beta^{\prime}$ non-conserved domain omitted for clarity). Colors as in B. 

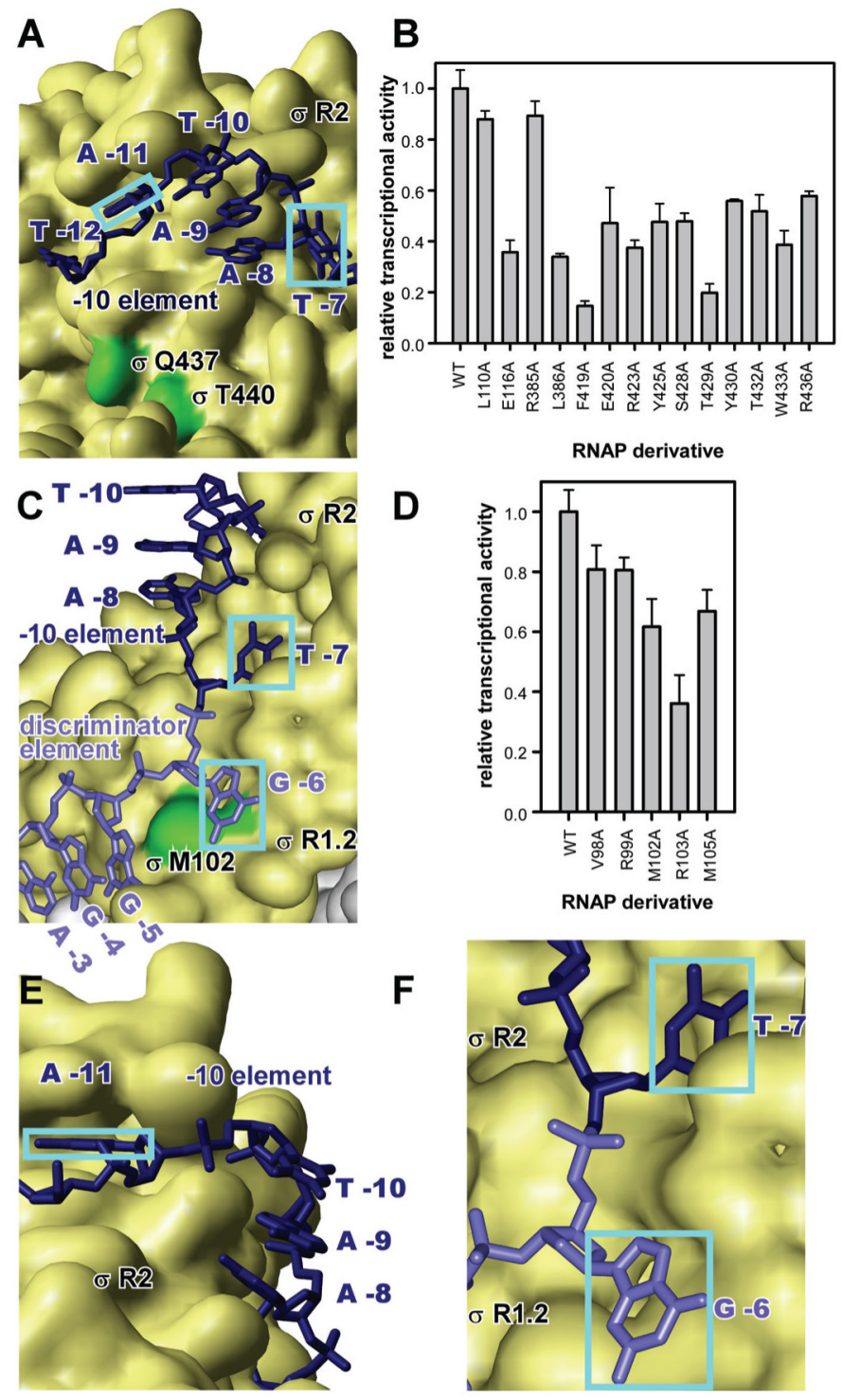

Fig. 2. Structure of RPo-GpA: recognition by $\sigma$ of $\mathbf{- 1 0}$ element and discriminator element (A) Interactions between $\sigma$ region $2(\sigma \mathrm{R} 2)$ and nontemplate-strand ssDNA of -10 element. Green, residues important for sequence recognition at position -12 (16-17; discussion in legend to Fig. S5). Other colors as in Fig. 1B-C. (B) Effects on transcription of substitutions of $\sigma$ residues that contact -10 -element bases. (C) Interactions between $\sigma$ region $1.2(\sigma \mathrm{R} 1.2)$ and nontemplate-strand ssDNA of discriminator element. Green, residue that crosslinks with position -5 (19). (D) Effects on transcription of substitutions of $\sigma$ residues that contact discriminator-element bases. (E) Recognition of A-11 base by unstacking and insertion into pocket formed by residues of $\sigma$. (F) Recognition of T-7 and G- 6 bases by unstacking and insertion into pocket formed by residues of $\sigma$. 

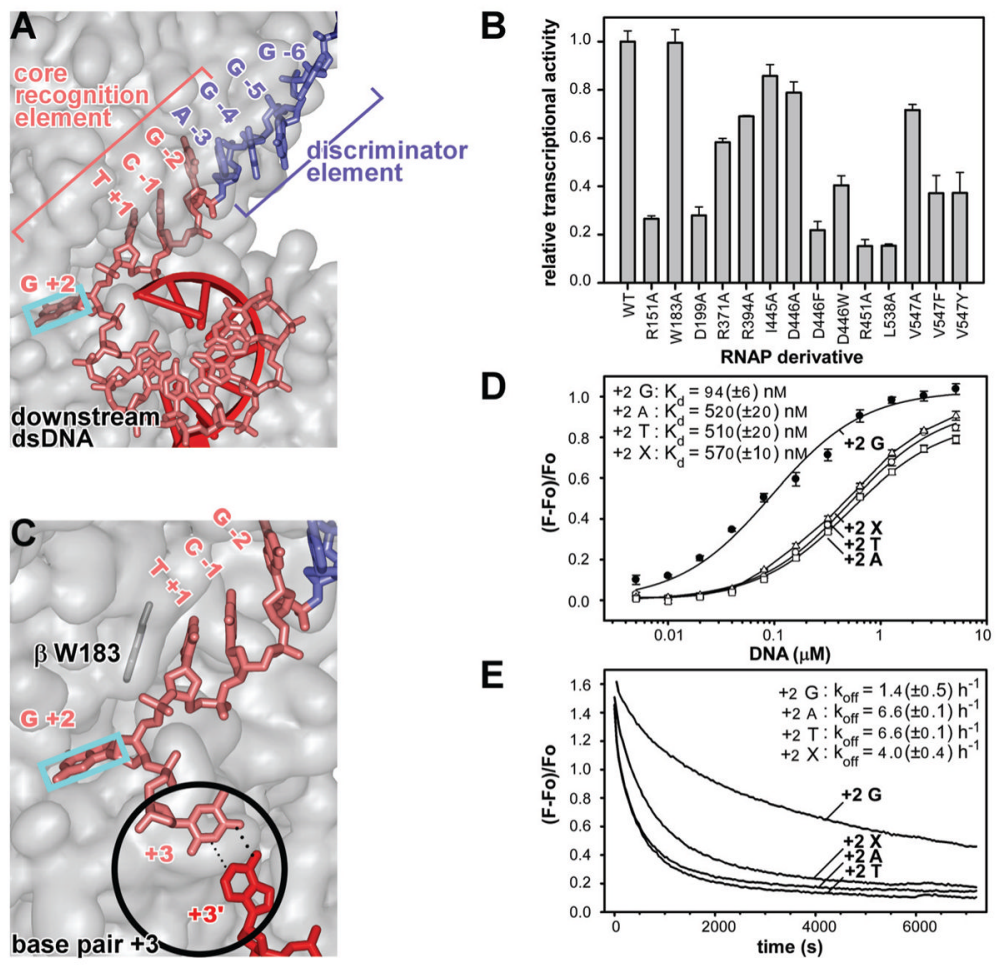

E

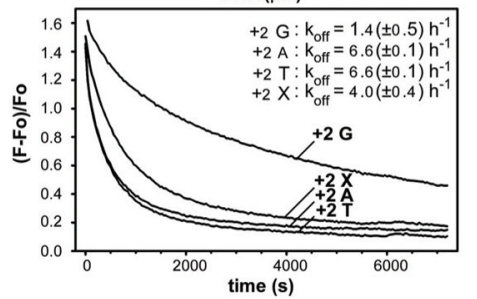

Fig. 3. Structure of RPo-GpA: recognition by RNAP of core recognition element (CRE) (A) Interactions between RNAP $\beta$ subunit and nontemplate-strand ssDNA of CRE (positions -4 through +2$)$. RNAP $\beta^{\prime}$ subunit and $\sigma$ omitted for clarity. Downstream dsDNA helix viewed end-on. Colors as in Fig. 1B-C. (B) Effects on transcription of substitutions of RNAP $\beta$-subunit residues that contact CRE bases. (C) Recognition of $\mathrm{G}+2$ base by unstacking and insertion into pocket formed by residues of RNAP $\beta$ subunit, and stacking of $\mathrm{T}+1$ base on RNAP $\beta$-subunit residue W183. (D),(E) Effects on RNAP-DNA interaction of substitutions of $\mathrm{G}+2$ base by $\mathrm{A}, \mathrm{T}$, or abasic site (X). Panel D shows fluorescence-detected equilibrium binding. Panel E shows fluorescence-detected high-salt-induced dissociation. 

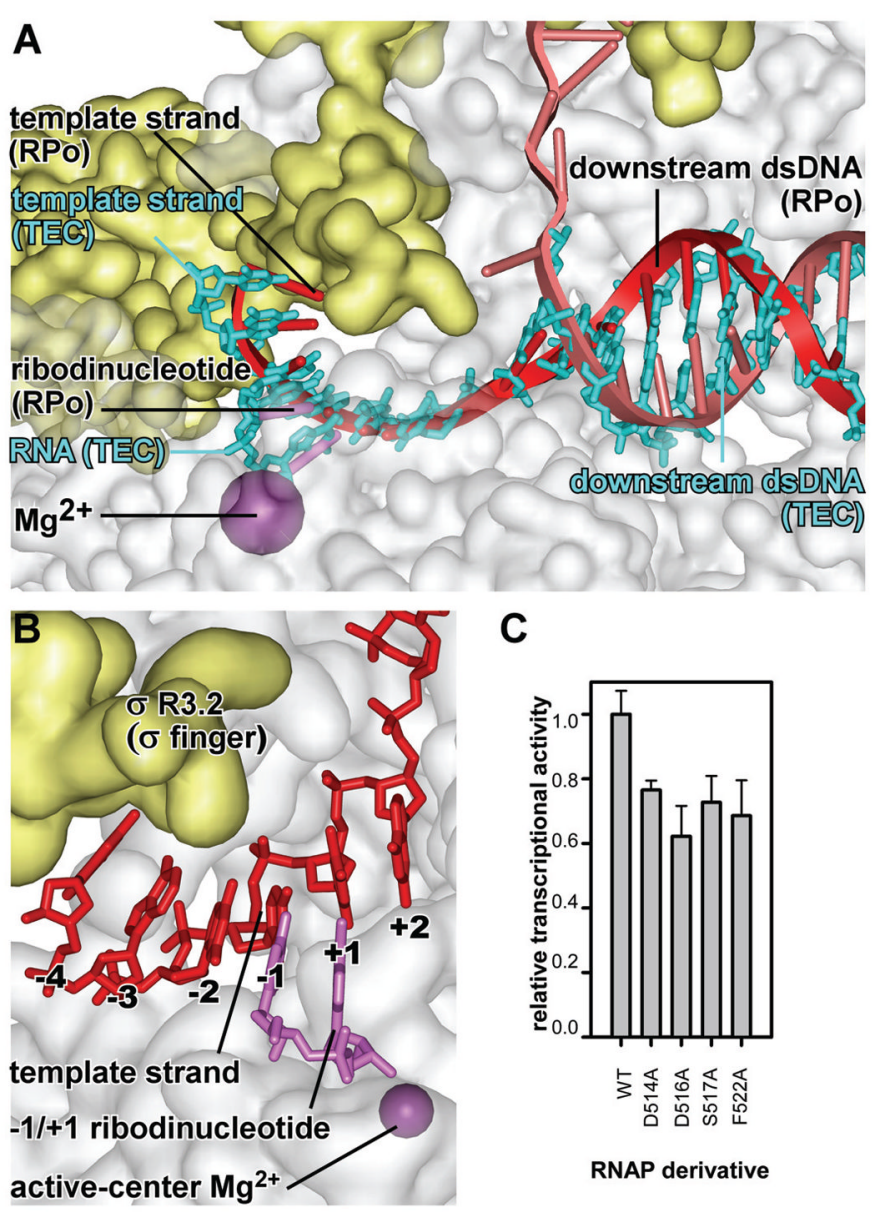

C
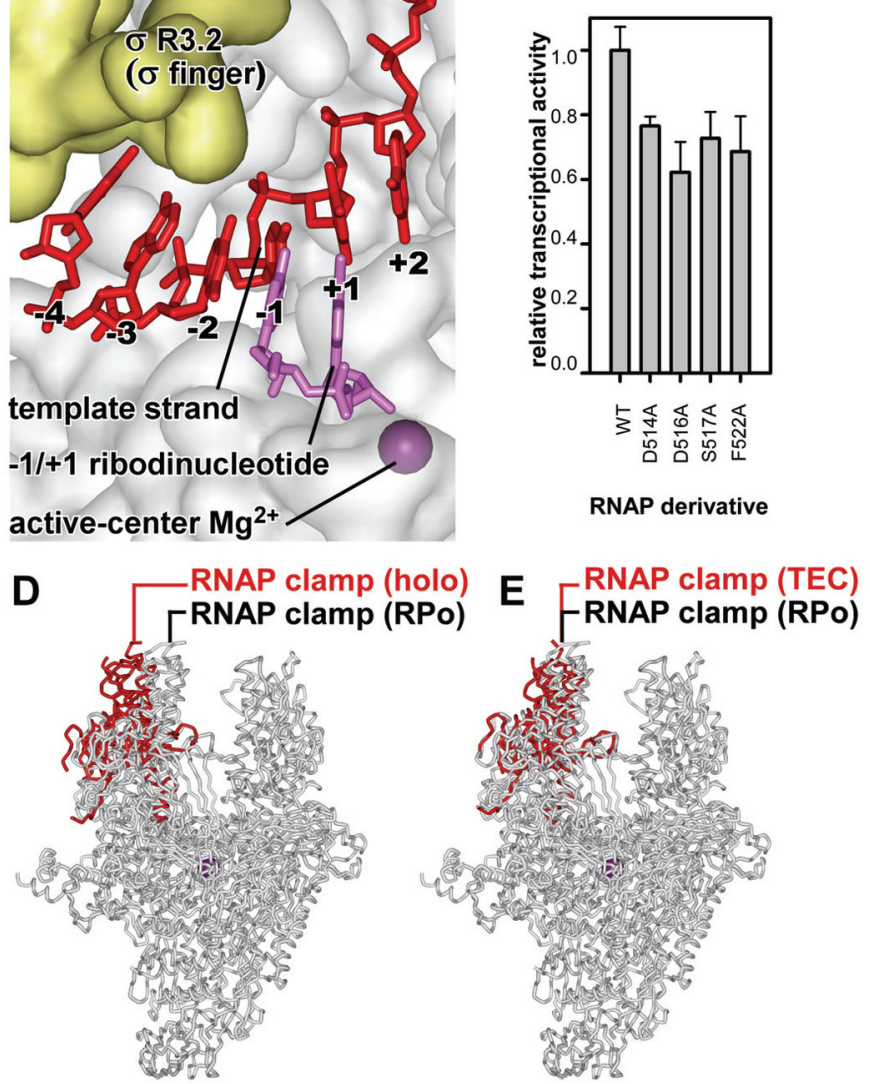

Fig. 4. Structure of RPo-GpA: pre-organization of transcription-bubble template-strand ssDNA, downstream dsDNA, and RNAP clamp

(A) Comparison of transcription-bubble template strand ssDNA, downstream dsDNA, and ribodinucleotide primer GpA in structure of RPo-GpA (colors as in Fig. 1B-C) with those in structure of $T$. thermophilus transcription elongation complex (29; cyan). (B) Interactions between $\sigma$ region 3.2 ( $\sigma \mathrm{R} 3.2$; " $\sigma$ finger") and transcription-bubble template-strand ssDNA. (C) Effects on transcription of substitutions of $\sigma$ residues that contact transcription-bubble template-strand bases. (D),(E) Comparison of RNAP clamp conformations in structures of RPo-GpA (colors as in Fig. 1B-C), T. thermophilus RNAP holoenzyme (30; red), and $T$. thermophilus transcription elongation complex (29; red). 